\title{
The Effect of the Timing of Dexamethasone Administration in Patients with COVID-19 Pneumonia
}

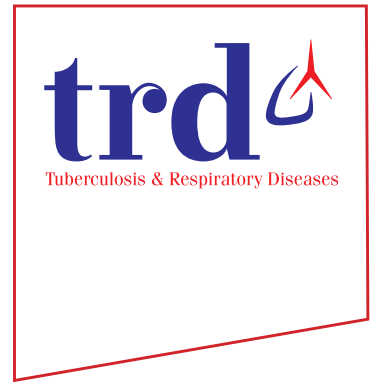

\author{
Hyun Woo Lee, M.D. ${ }^{\circledR}$, Jimyung Park, M.D., Jung-Kyu Lee, M.D., Tae Yeon Park, M.D. and Eun \\ Young Heo, M.D.
}

Division of Respiratory and Critical Care, Department of Internal Medicine, Seoul Metropolitan Government-Seoul National University Boramae Medical Center, Seoul, Republic of Korea

Background: Despite the proven benefits of dexamethasone in hospitalized coronavirus disease 2019 (COVID-19) patients, the optimum time for the administration of dexamethasone is unknown. We investigated the progression of COVID-19 pneumonia based on the timing of dexamethasone administration.

Methods: A single-center, retrospective cohort study based on medical record reviews was conducted between June 10 and September 21, 2020. We compared the risk of severe COVID-19, defined as the use of a high-flow nasal cannula or a mechanical ventilator, between groups that received dexamethasone either within 24 hours of hypoxemia (early dexamethasone group) or 24 hours after hypoxemia (late dexamethasone group). Hypoxemia was defined as room-air $\mathrm{SpO}_{2}<90 \%$.

Results: Among 59 patients treated with dexamethasone for COVID-19 pneumonia, 30 were in the early dexamethasone group and 29 were in the late dexamethasone group. There was no significant difference in baseline characteristics, the time interval from symptom onset to diagnosis or hospitalization, or the use of antiviral or antibacterial agents between the two groups. The early dexamethasone group showed a significantly lower rate of severe COVID-19 compared to the control group $(75.9 \%$ vs. $40.0 \%$, $\mathrm{p}=0.012)$. Further, the early dexamethasone group showed a significantly shorter total duration of oxygen supplementation (10.45 days vs. 21.61 days, $\mathrm{p}=0.003)$ and length of stay in the hospital (19.76 days vs. 27.21 days, $\mathrm{p}=0.013$ ). However, extracorporeal membrane oxygenation and in-hospital mortality rates were not significantly different between the two groups.

Conclusion: Early administration of dexamethasone may prevent the progression of COVID-19 to a severe disease, without increased mortality.

Keywords: Coronavirus Disease 2019 (COVID-19); Pneumonia; Inhalation Therapy, Oxygen; Dexamethasone; Respiratory Failure

Address for correspondence: Eun Young Heo, M.D.

Department of Internal Medicine, Seoul Metropolitan Government-Seoul National University Boramae Medical Center, 20 Boramae-ro 5-gil, Dongjakgu, Seoul 07061, Republic of Korea

Phone: 82-2-870-3207, Fax: 82-2-831-0714, E-mail: eunyoungheo@gmail.com

Received: Jan. 18, 2021, Revised: Mar. 9, 2021, Accepted: Mar. 28, 2021, Published online: Mar. 29, 2021

@(t) is identical to the Creative Commons Attribution Non-Commercial License (http://creativecommons.org/licenses/by-nc/4.0/). 


\section{Introduction}

Systemic administration of corticosteroids has been associated with decreased in-hospital mortality in coronavirus disease 2019 (COVID-19) patients with hypoxemia ${ }^{1}$. Among systemic corticosteroids, dexamethasone has been demonstrated to improve mortality in COVID-19 patients in two randomized controlled trials (RCTs) ${ }^{2,3}$, but the benefit of hydrocortisone or methylprednisolone is not as clear ${ }^{1,4,5}$. However, $36.2 \%$ (166/459) of patients who used dexamethasone eventually died ${ }^{1}$, suggesting that dexamethasone should be used in a timely manner only in selective patients ${ }^{6}$. Nevertheless, there is little information concerning the prognosis for COVID-19 patients that relates to different times of dexamethasone administration.

The efficacy of dexamethasone in protecting against acute respiratory distress syndrome (ARDS) is likely to be related to the time of initial drug administration. A recent RCT showed that dexamethasone administration within 30 hours of ARDS onset decreased the duration of mechanical ventilation and mortality rates ${ }^{7}$. COVID-19 studies also showed a potential benefit of early dexamethasone treatment in improving the prognosis in patients with acute respiratory failure. In the CoDEX trial, dexamethasone administration within 48 hours of the onset of ARDS significantly increased ventilator-free days in the COVID-19 patients ${ }^{3}$. A quasi-experimental study of moderate-to-severe COVID-19 cases found that systemic corticosteroid administered from the first day of oxygen supplementation reduced a composite of primary outcomes, including intensive care unit care, progression to respiratory failure, and in-hospital mortality ${ }^{8}$. In addition, there was a positive correlation between early corticosteroid treatment and a better clinical prognosis in severe acute respiratory syndrome ${ }^{9}$.

The timing of dexamethasone administration is of special concern in those COVID-19 patients who need only oxygen therapy. This study determined whether there is a difference in the rate of ARDS progression, based on the time of dexamethasone administration, in COVID-19 patients who needed only oxygen therapy without either high-flow nasal cannula (HFNC) therapy or a mechanical ventilator (MV).

\section{Materials and Methods}

Our study was reported in accordance with the guidelines of the Strengthening the Reporting of Observational Studies in Epidemiology (STROBE) statement ${ }^{10}$.

\section{Study design and participants}

The present retrospective cohort study included all the hospitalized patients who received dexamethasone for COVID-19 pneumonia at Seoul Metropolitan Government-Seoul
National University Boramae Medical Center (SMG-SNU BMC), Seoul, between June 10 and September 21, 2020. A diagnosis of COVID-19 was confirmed through quantitative reverse transcription-polymerase chain reaction (RT-qPCR) assays using upper or lower respiratory tract samples.

Patients were admitted to the isolation ward for COVID-19 management, which has negative pressure ventilation. Dexamethasone was administrated to the COVID-19 patients who required oxygen therapy or had a significant desaturation (a change in pulse oximeter oxygen saturation $\left[\mathrm{SpO}_{2}\right]$ $>4 \%$ ) with clinical signs of aggravated pneumonia. The time of dexamethasone administration was determined by the attending physician. Oxygen supplementation was initiated when patients had a room-air $\mathrm{SpO}_{2}<90 \%$, which is defined as hypoxemia. Oxygen saturation was monitored through pulse oximetry for 24 hours during oxygen supplementation. Administration of remdesivir was allowed in the patients who met all of the following criteria: (1) pneumonic infiltration was evident in a chest X-ray or chest computed tomography (CT) scan, (2) room-air $\mathrm{SpO}_{2}<94 \%$, (3) oxygen supplementation, and $(4) \leq 10$ days since symptom onset. In practice, remdesivir was administered only to patients over 70 years of age because of the limited supply of remdesivir during the study period. Administration of antibiotics for respiratory infections was also allowed when a bacterial infection had not been ruled out.

\section{Eligibility criteria}

The eligibility criteria were as follows: (1) age $>18$ years old, (2) detection of severe acute respiratory syndrome coronavirus 2 by an RT-qPCR assay of upper or lower respiratory sample, (3) evidence of pneumonia in a chest X-ray or chest CT scan, and (4) administration of dexamethasone before initiation of HFNC treatment or a MV. Exclusion criteria were immunosuppressive diseases, treatment with a systemic corticosteroid or other immunosuppressive drugs, pregnancy, terminal-stage cancer, or other end-stage diseases.

\section{Variables and measurements}

Demographic information for the study population was collected, including age, sex, body mass index, smoking status, Charlson comorbidity index (CCI), and underlying diseases (hypertension, diabetes, cerebrovascular disease, cardiovascular disease, chronic lung disease, chronic kidney disease, chronic liver disease, and cancer). Symptoms of COVID-19 were determined by medical staff when dexamethasone treatment was initiated, including abnormal senses of smell or taste, myalgia, sore throat, cough, sputum, chest discomfort, dyspnea, fever or chills, rhinorrhea, and nausea or diarrhea. Ct-values of RT-qPCR at first diagnosis were obtained. Clinical severity was evaluated using the Sequential Organ Failure As- 
sessment (SOFA) score and the Simplified Acute Physiology Score (SAPS) II, $\mathrm{SpO}_{2}$, and $\mathrm{PaO}_{2} / \mathrm{FiO}_{2}$ ratio (ratio of arterial partial pressure of oxygen to fraction of inspired oxygen) at hypoxemia development. Laboratory tests included assays for white blood cells, lymphocytes, C-reactive protein, procalcitonin, lactate dehydrogenase, troponin-I, and D-dimer. Treatment information included the use of antiviral or antibacterial agents, total dose of dexamethasone, duration of dexamethasone treatment, the time interval from symptom onset or diagnosis to initiation of dexamethasone administration, and the time interval from hypoxemia to dexamethasone administration.

\section{Study groups and outcomes}

We defined the initiation time of dexamethasone treatment as the time interval from occurrence of hypoxemia to initiation of dexamethasone treatment. The distribution of the initiation time of dexamethasone was largely divided into two groups based on the criteria of 24 hours in a pilot analysis. The patients with the initiation time of dexamethasone treatment $<24$ hours were defined as early dexamethasone group and those $\geq 24$ hours were defined as late dexamethasone group. The primary outcome was to compare the rate of HFNC or MV treatments between early and late dexamethasone group. HFNC was used when oxygen supplementation with nasal prong $6 \mathrm{~L} / \mathrm{min}$ or more was required. The secondary outcome was to compare the total duration of oxygen supplementation and the length of stay in hospital for the two groups.

\section{Statistical analyses}

Demographic information, symptoms, clinical features, clinical severity, and study outcomes were compared between the early and late dexamethasone groups. We conducted ShapiroWilk normality test for each continuous variable before statistical tests. Categorical variables were analyzed with Pearson's chi-square test or the Fisher exact test. Continuous variables were analyzed with the Student's t test or Mann-Whitney U test. Repeated measure analysis of variance was conducted for analysis of serial physiologic parameters (respiratory rate, heart rate, and $\mathrm{SpO}_{2}$ ) and laboratory results (complete blood count). Logistic regression analysis was used to determine whether the timing of dexamethasone administration correlated with HFNC or MV treatments by calculating odds ratios and $95 \%$ confidence intervals (CIs). To exclude potential bias due to confounding factors associated with the use of HFNC or MV treatments, we conducted a multivariate analysis. Independent variables were selected on the basis of their statistical significance in the univariate analysis. The criterion for inclusion of a variable in the multivariate analysis was based on the clinical significance. The Kaplan-Meier curve was used to visualize the difference in the time to HFNC or MV treatment between the early and late dexamethasone groups, and the statistical significance was estimated by the log-rank test. Univariable and multivariable Cox regression analyses were used to evaluate hazard ratios (HRs) for prolonged time to HFNC or MV treatments. Multivariable analyses were conducted using two models. In model 1, early dexamethasone group was used as a categorical variable for multivariable analysis. In model 2, initiation time of dexamethasone treatment was used as a continuous variable for multivariable analysis. We considered p-values $<0.05$ as statistically significant. All the statistical analyses were conducted using $R$ statistical software (version 3.5.1, 2018, R Core Team, Vienna, Austria).

\section{Ethics}

The Institutional Review Board Committee of Seoul National University Seoul Metropolitan Government (SNUSMG) Boramae Medical Center approved the study protocol and waived the need for informed consent for access to the electronic medical records (IRB No. 20-2020-33).
212 Patients who were hospitalized for SARS-CoV-2 confirmed by PCR in Boramae Medical Center from June 10 to September 21, 2020

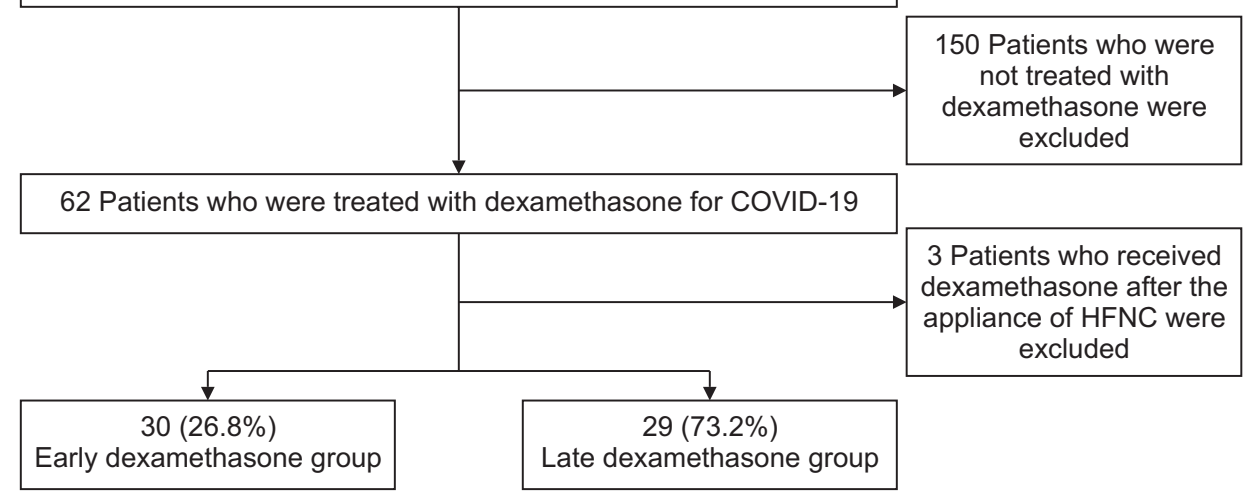

Figure 1. Flow chart of patient inclusion. SARS-CoV-2: severe acute respiratory syndrome coronavirus 2; PCR: polymerase chain reaction; COVID-19: coronavirus disease 2019; HFNC: high-flow nasal cannula. 


\section{Results}

Among a total of 212 patients hospitalized for treatment of COVID-19, 62 (29.2\%) were treated with dexamethasone. After excluding three patients who received dexamethasone after HFNC treatment, 59 patients $(27.8 \%)$ were placed in two groups: an early dexamethasone group (30 patients) and a late dexamethasone group (29 patients) (Figure 1). Time interval from hypoxemia to dexamethasone administration was median 0 day (interquartile range [IQR], $0-0$ ) in early dexamethasone group and median 2 days (IQR, 1-2) in late dexamethasone group. The time interval from symptom onset to diagnosis of COVID-19 was a median 3.0 days (IQR, $0-5.0$ ). The median time interval from symptom onset to hospitaliza- tion for COVID-19 was 3.0 days (IQR, 1.0-6.5). All the patients showed evidence of pneumonic infiltration in the initial chest $\mathrm{X}$-ray at admission.

\section{Baseline characteristics and clinical features of the study population}

In baseline characteristics, age, percentage of current smokers, and CCI were higher in the late dexamethasone group, but the values were not statistically significant (Table 1). The frequency of each symptom was not significantly different between the early and late dexamethasone groups.

In terms of clinical features, early dexamethasone group showed a higher SOFA score and SAPS II at hypoxemia devel-

Table 1. Baseline characteristics of COVID-19 patients in early and late dexamethasone groups

\begin{tabular}{|lccc|}
\hline \multicolumn{1}{c}{ Characteristic } & $\begin{array}{c}\text { Late dexamethasone group } \\
(\mathbf{n}=\mathbf{2 9})\end{array}$ & $\begin{array}{c}\text { Early dexamethasone group } \\
(\mathbf{n}=\mathbf{3 0})\end{array}$ & p-value \\
\hline Age, yr & $70.07 \pm 13.87$ & $65.53 \pm 14.39$ & 0.138 \\
\hline Female sex & $12(41.4)$ & $10(33.3)$ & 0.712 \\
\hline Body mass index & $24.32 \pm 4.64$ & $24.68 \pm 4.33$ & 0.764 \\
\hline Current smoker & $5(17.2)$ & $2(6.7)$ & 0.394 \\
\hline Charlson comorbidity index & $4.34 \pm 2.06$ & $3.50 \pm 2.35$ & 0.147 \\
\hline Underlying disease & & & $>0.990$ \\
\hline Hypertension & $15(51.7)$ & $15(50.0)$ & 0.928 \\
\hline Diabetes mellitus & $10(34.5)$ & $9(30.0)$ & 1.000 \\
\hline Cerebrovascular disease & $4(13.8)$ & $5(16.7)$ & 0.465 \\
\hline Cardiovascular disease & $7(24.1)$ & $4(13.3)$ & 0.666 \\
\hline Chronic lung disease & $5(17.2)$ & $3(10.0)$ & 0.449 \\
\hline Chronic kidney disease & $2(6.9)$ & $5(16.7)$ & $>0.990$ \\
\hline Chronic liver disease & $3(10.3)$ & $3(10.0)$ & 0.394 \\
\hline Cancer & $5(17.2)$ & $2(6.7)$ & $>0.990$ \\
\hline Symptoms & & & 0.712 \\
\hline Abnormality in sense of smell and taste & $2(6.9)$ & $2(6.7)$ & 0.503 \\
\hline Myalgia & $12(41.4)$ & $10(33.3)$ & 0.516 \\
\hline Sore throat & $3(10.3)$ & $6(20.0)$ & 0.358 \\
\hline Cough & $16(55.2)$ & $13(43.3)$ & 0.629 \\
\hline Sputum & $12(41.4)$ & $8(26.7)$ & 0.502 \\
\hline Chest discomfort & $1(3.4)$ & $3(10.0)$ & 0.244 \\
\hline Dyspnea & $18(62.1)$ & $15(50.0)$ & $>0.990$ \\
\hline Febrile or chilling sense & $14(48.3)$ & $20(66.7)$ & 0.962 \\
\hline Rhinorrhea or nasal obstruction & $0(0)$ & $1(3.3)$ & 0.968 \\
\hline Gastrointestinal symptoms & $4(13.8)$ & $3(10.0)$ & $2(6.7)$ \\
\hline No symptoms & $3(10.3)$ & & \\
\hline
\end{tabular}

Values are presented as the mean \pm standard deviation or number (\%).

COVID-19: coronavirus disease 2019. 
Table 2. Clinical features and outcomes of COVID-19 patients in early and late dexamethasone groups

\begin{tabular}{|c|c|c|c|}
\hline Variable & $\begin{array}{l}\text { Late dexamethasone group } \\
\qquad(\mathrm{n}=29)\end{array}$ & $\begin{array}{l}\text { Early dexamethasone group } \\
\qquad(\mathrm{n}=30)\end{array}$ & p-value \\
\hline \multicolumn{4}{|l|}{ Clinical features } \\
\hline \multicolumn{4}{|l|}{ Ct-value of RT-PCR at first diagnosis } \\
\hline Egene & $22.07 \pm 6.32$ & $21.65 \pm 4.92$ & 0.786 \\
\hline RdRP gene & $21.80 \pm 6.49$ & $20.36 \pm 5.60$ & 0.382 \\
\hline \multicolumn{4}{|l|}{ Clinical severity } \\
\hline SOFA score at hypoxemia development & $3.5 \pm 1.8$ & $4.6 \pm 2.2$ & 0.044 \\
\hline SAPS II at hypoxemia development & $21.1 \pm 6.2$ & $26.7 \pm 9.7$ & 0.010 \\
\hline $\mathrm{SpO}_{2}$ at hypoxemia development & $88.9 \pm 4.9$ & $87.0 \pm 3.1$ & 0.081 \\
\hline $\mathrm{PaO}_{2} / \mathrm{FiO}_{2}$ ratio at hypoxemia development & $296 \pm 117$ & $202 \pm 63$ & $<0.001$ \\
\hline \multicolumn{4}{|l|}{ Laboratory test at hypoxemia development } \\
\hline White blood cell counts, $\times 10^{3} / \mu \mathrm{L}$ & $5,963 \pm 3,705$ & $6,490 \pm 2,377$ & 0.517 \\
\hline The number of lymphocytes, $\times 10^{3} / \mu \mathrm{L}$ & $946 \pm 376$ & $1,018 \pm 499$ & 0.533 \\
\hline C-reactive protein, $\mathrm{mg} / \mathrm{dL}$ & $7.50 \pm 6.34$ & $9.59 \pm 6.50$ & 0.217 \\
\hline Procalcitonin, ng/mL & $0.05(0.04-0.08)$ & $0.09(0.05-0.21)$ & 0.181 \\
\hline Lactate dehydrogenase, IU/L & $293(220-376)$ & $367(303-450)$ & 0.111 \\
\hline Troponin-I, ng/mL & $9.1(4.2-13.8)$ & $10.2(5.2-19.2)$ & 0.124 \\
\hline D-dimer, mg/L & $0.8(0.5-2.1)$ & $0.8(0.6-1.6)$ & 0.640 \\
\hline \multicolumn{4}{|l|}{ Treatment } \\
\hline Remdesivir & $13(44.8)$ & $12(40.0)$ & 0.911 \\
\hline Antibiotics & $29(100)$ & $30(100)$ & NA \\
\hline Total accumulative dose of dexamethasone mg & $62.62 \pm 33.34$ & $64.17 \pm 32.48$ & 0.857 \\
\hline Total duration of dexamethasone mg & $10.66 \pm 5.58$ & $10.57 \pm 4.48$ & 0.947 \\
\hline $\begin{array}{l}\text { Time interval from symptom onset to dexamethasone } \\
\text { administration, day }\end{array}$ & $7.76 \pm 3.59$ & $7.03 \pm 3.50$ & 0.435 \\
\hline $\begin{array}{l}\text { Time interval from diagnosis to dexamethasone } \\
\text { administration, day }\end{array}$ & $4.14 \pm 3.26$ & $4.70 \pm 3.67$ & 0.537 \\
\hline $\begin{array}{l}\text { Time interval from hypoxemia to dexamethasone } \\
\text { administration, day }\end{array}$ & $2(1-2)$ & $0(0-0)$ & $<0.001$ \\
\hline \multicolumn{4}{|l|}{ Clinical outcomes } \\
\hline High-flow nasal cannula or mechanical ventilation & $22(75.9)$ & $12(40.0)$ & 0.012 \\
\hline Extracorporeal membrane oxygenation & $2(6.9)$ & $0(0)$ & 0.457 \\
\hline Total duration of oxygen supplementation ${ }^{*, \dagger}$, day & $21.61 \pm 16.42$ & $10.45 \pm 9.39$ & 0.003 \\
\hline Length of stay in hospital ${ }^{\dagger}$, day & $27.21 \pm 13.28$ & $19.76 \pm 8.05$ & 0.013 \\
\hline In-hospital death & $1(3.4)$ & $1(3.3)$ & $>0.990$ \\
\hline
\end{tabular}

Values are presented as the mean \pm standard deviation, median (interquartile range), or number (\%).

*Oxygen supplementation includes oxygen inhalation through nasal prong, facial mask, high-flow nasal cannula, mechanical ventilator, and extracorporeal membrane oxygenation. ${ }^{\dagger}$ Data regarding total duration of oxygen supplementation and length of stay in hospital were missing for 1 patient in early dexamethasone group and 1 patient in late dexamethasone group.

COVID-19: coronavirus disease 2019; RT-PCR: reverse transcription polymerase chain reaction; SOFA: Sequential Organ Failure Assessment; SAPS: Simplified Acute Physiology Score; $\mathrm{SpO}_{2}$ : pulse oximeter oxygen saturation; $\mathrm{PaO}_{2} / \mathrm{FiO}_{2}$ ratio: ratio of arterial partial pressure of oxygen to fraction of inspired oxygen. 
opment (Table 2). $\mathrm{PaO}_{2} / \mathrm{FiO}_{2}$ ratio was lower in the early dexamethasone group. While heart rate serially decreased, $\mathrm{SpO}_{2}$, applied oxygen flow rate, white blood cells, and platelet were increased during initial 3 days after dexamethasone administration (Supplementary Table S1). In terms of treatments, the rate of remdesivir use or antibiotic use was very similar in the early and late dexamethasone groups. The dose of dexamethasone and the duration of dexamethasone treatment were similar between the two groups. Two patients in the early dexamethasone group started dexamethasone $1.5( \pm 0.7)$ days before oxygen supplementation because they showed imminent hypoxemic respiratory failure with fever, a progression of pneumonic infiltration, and decreasing $\mathrm{SpO}_{2}$.

\section{Association between clinical outcomes and the time of dexamethasone administration}

Twelve patients (40.0\%) in the early dexamethasone group and 22 patients $(75.9 \%)$ in the late dexamethasone group required HFNC or MV treatment, which was significantly different between the two groups ( $\mathrm{p}=0.012$ ) (Table 2). MV was applied to seven patients (23.3\%) in early dexamethasone group and 13 patients (44.8\%) in late dexamethasone group $(\mathrm{p}=0.142)$. Two patients in the late dexamethasone group were given extracorporeal membrane oxygenation (ECMO) and there was no difference in ECMO use between the two groups. The early dexamethasone group showed a significant- ly shorter duration of oxygen supplementation (10.45 days vs. 21.61 days, $\mathrm{p}=0.003$ ) and length of stay in hospital (19.76 days vs. 27.21 days, $p=0.013$ ). We found no difference in in-hospital deaths between the two groups, which were caused in both groups by respiratory failure.

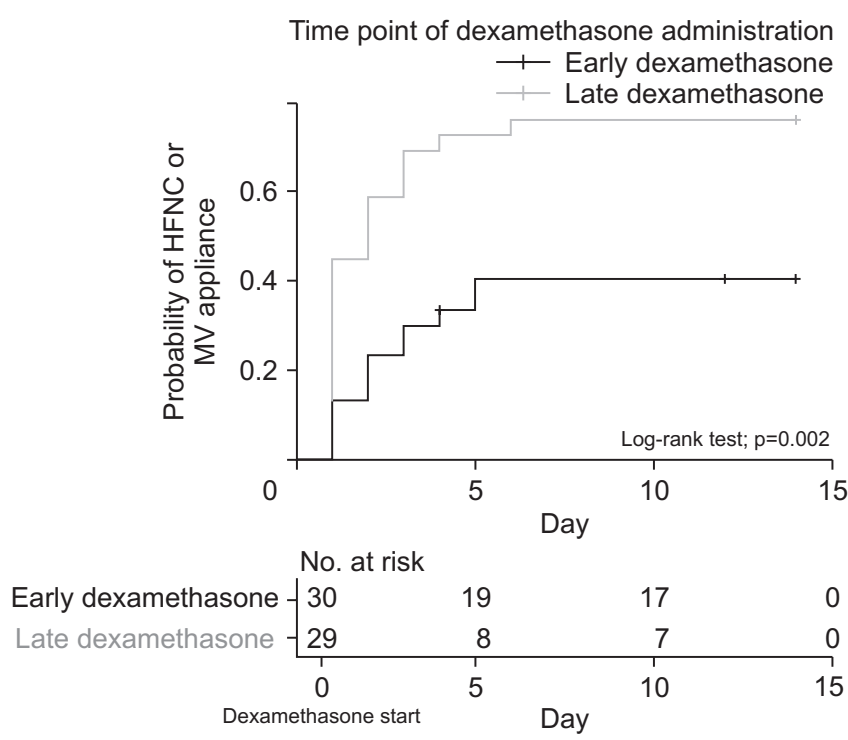

Figure 2. Kaplan-Meier curve for probability of high-flow nasal cannula (HFNC) or mechanical ventilator (MV) treatment for 2 weeks after the initiation of dexamethasone administration.

Table 3. Cox regression analyses to evaluate the probability for high-flow nasal cannula or mechanical ventilation according to time point of dexamethasone administration

\begin{tabular}{|c|c|c|c|c|c|c|c|c|c|}
\hline \multirow[t]{2}{*}{ Variable } & \multicolumn{2}{|c|}{ Univariable analysis } & \multirow{2}{*}{ p-value } & \multicolumn{2}{|c|}{$\begin{array}{l}\text { Multivariable analysis } \\
\text { (model 1) }\end{array}$} & \multirow{2}{*}{ p-value } & \multicolumn{2}{|c|}{$\begin{array}{c}\text { Multivariable analysis } \\
\text { (model 2) }\end{array}$} & \multirow[t]{2}{*}{ p-value } \\
\hline & HR & $95 \%$ CI & & HR & $95 \%$ CI & & HR & $95 \% \mathrm{CI}$ & \\
\hline Age & 1.020 & $0.996-1.045$ & 0.101 & - & - & - & - & - & - \\
\hline Female sex & 0.655 & $0.319-1.344$ & 0.249 & 1.142 & $0.499-2.613$ & 0.753 & 0.945 & $0.417-2.144$ & 0.893 \\
\hline Current smoker & 2.448 & $1.009-5.944$ & 0.048 & 2.071 & $0.726-5.903$ & 0.173 & 1.310 & $0.421-4.073$ & 0.641 \\
\hline Charlson comorbidity index & 1.147 & $0.977-1.347$ & 0.095 & 1.032 & $0.850-1.253$ & 0.750 & 0.976 & $0.805-1.183$ & 0.803 \\
\hline SOFA score & 1.245 & $1.101-1.409$ & $<0.001$ & 1.182 & $0.992-1.408$ & 0.062 & 1.157 & $0.955-1.402$ & 0.135 \\
\hline SAPS II & 1.089 & $1.049-1.131$ & $<0.001$ & - & - & - & - & - & - \\
\hline $\begin{array}{l}\text { No. of lymphocytes at } \\
\text { development of hypoxemia }\end{array}$ & 0.999 & $0.998-1.000$ & 0.036 & 0.999 & $0.998-1.000$ & 0.023 & 0.999 & $0.998-1.000$ & 0.034 \\
\hline Remdesivir & 2.358 & $1.184-4.695$ & 0.015 & 1.733 & $0.847-3.546$ & 0.133 & 1.544 & $0.721-3.308$ & 0.264 \\
\hline Early dexamethasone & 0.344 & $0.169-0.698$ & 0.003 & 0.440 & $0.211-0.915$ & 0.028 & - & - & - \\
\hline $\begin{array}{l}\text { Initiation time of } \\
\text { dexamethasone treatment }\end{array}$ & 1.139 & $0.902-1.439$ & 0.275 & - & - & - & 1.180 & $0.880-1.582$ & 0.268 \\
\hline
\end{tabular}

Values are presented as the odds ratio and $95 \%$ confidence interval.

In model 1, early dexamethasone (initiation time of dexamethasone treatment within 24 hours) was used as a categorical variable for multivariable analysis. In model 2, initiation time of dexamethasone treatment was used as a continuous variable for multivariable analysis. multivariable dox regression analysis was adjusted by covariates including SOFA score and SAPS II.

Age and SAPS II are not included in multivariable analysis due to multicollinearity (variance inflation factor $>4$ ).

HR: hazard ratio; CI: confidence interval; SOFA: Sequential Organ Failure Assessment; SAPS: Simplified Acute Physiology Score. 
In the Kaplan-Meir estimate, the probability of HFNC or MV treatment was significantly lower in the early dexamethasone group (log-rank test=0.002) (Figure 2). Univariable Cox regression analysis revealed that the probability of HFNC or MV treatment was significantly lower over time in the early dexamethasone group (HR, 0.344 [95\% CI, 0.169-0.698]; p=0.003) (Table 3). However, initiation time of dexamethasone treatment was not significantly associated with the probability of HFNC or MV treatment. In the multivariable Cox regression analysis, the probability of HFNC or MV treatment was significantly lower in the early dexamethasone group (HR, 0.440 [95\% CI, 0.211-0.915]; $\mathrm{p}=0.028$; model 1), but not significantly related with initiation time of dexamethasone treatment (model 2).

\section{Discussion}

Our study identified a correlation between the administration of dexamethasone within 24 hours of oxygen supplementation (early dexamethasone) and a lower rate of HFNC or MV treatment in COVID-19 patients who required oxygen therapy or had a significant desaturation. In addition, the early dexamethasone group showed a significantly shorter total duration of oxygen supplementation and length of stay in hospital. Early dexamethasone treatment was significantly correlated with a lower rate of HFNC or MV treatment, even in multivariable Cox regression analysis. However, the probability of HFNC or MV treatment was not associated with the initiation time of dexamethasone in Cox regression analyses, which finding suggests that the risk of HFNC or MV treatment was not linearly increased as the initiation time of dexamethasone was prolonged. Although the early dexamethasone group required less HFNC or MV treatment, there was no difference in ECMO use or in-hospital mortality rates between the early and late dexamethasone groups. Therefore, early dexamethasone administration for hypoxemic COVID-19 patients may reduce the need for HFNC or MV treatment without increased mortality; thus, reducing the need for scarce medical resources in the COVID-19 pandemic ${ }^{11}$.

Although we know that dexamethasone is associated with a lower mortality rate in COVID-19 patients undergoing oxygen therapy alone ${ }^{2}$, information is needed about when dexamethasone treatment should be initiated to maximize the benefits. Considering the evidence to date, the administration of corticosteroids seems to be more effective for COVID-19 patients with active progression to ARDS than those with early-phase pneumonia. In the RECOVERY study, dexamethasone reduced more mortality in the invasive MV group (25.2\%; rate ratio $=0.64[95 \% \mathrm{CI}, 0.51-0.81])$, in which pneumonia was more advanced, than the oxygen-only group $(37.5 \%$; rate ratio, 0.82 [95\% CI, 0.72-0.94] $)^{2}$. In the CoDEX study, dexamethasone benefited COVID-19 patients who used an MV after ARDS developed ${ }^{3}$. A recently published meta-analysis showed that dexamethasone benefits a subgroup of patients with symptom onset $>7$ days ${ }^{1}$. The mechanism by which dexamethasone reduces the pulmonary and systematic inflammatory process may contribute to these results ${ }^{12}$.

The progression of COVID-19 pneumonia from mild to severe disease can be characterized by initial mild infection phase followed by pulmonary inflammation phase, and then systemic hyper-inflammation phase ${ }^{13,14}$. Down-regulation of pulmonary and systemic inflammation is considered one of the most decisive factors for restoration to normal physiology in COVID-19 pneumonia ${ }^{15}$. Therapeutic strategy for ARDS due to COVID-19 should be based on suppression of pathological inflammation by controlling macrophage activation and cytokine release $\mathrm{e}^{16}$. The clinical benefits of glucocorticoid in ARDS have been explained by improved profiles of biologic markers for alveolar-capillary membrane permeability and mediators for inflammation and tissue repair ${ }^{17}$. In RCTs that proved the efficacy of dexamethasone in ARDS, dexamethasone treatment was initiated in the early phase of ARDS (within 48 hours of onset) $)^{3,7}$. Therefore, we can speculate that early administration of dexamethasone may attenuate the extent of lung injury or systemic organ damage and prevent progression to severe ARDS or systemic cytokine storm.

In our study, the number of patients in the early and late dexamethasone treatment groups was almost the same and two groups were distributed relatively evenly over study period, which reflects the controversy in clinical practice concerning the optimum time for dexamethasone administration. In the late dexamethasone group, it is likely that the physician did not expect the oxygen demand of the patient to increase; thus, dexamethasone would not have been be prescribed immediately. In fact, before studies on mortality reduction by dexamethasone were published, we had COVID-19 pneumonia cases where the oxygen demand gradually decreased without dexamethasone ${ }^{18}$. However, there is still no good tool to predict whether COVID-19 pneumonia will worsen. Serious adverse events were no different between patients treated or not treated with dexamethasone, while the benefit of dexamethasone was significant only in the oxygen group ${ }^{1,2}$. In our study, dexamethasone treatment within 24 hours of oxygen supplementation decreased the need for HFNC or MV interventions. The mean time from symptom onset to dexamethasone treatment was about 7.4 days. Therefore, when oxygen supplementation is needed, especially after 7 days of symptom onset, prompt administration of dexamethasone may be advantageous.

The present study has several limitations. First, it should be noted that there is a risk of bias in generalizing our results to all the patients with COVID-19 pneumonia. This study was a single-center retrospective observational study with a small number of patients. Our study population was highly selective by narrow range of inclusion criteria and confounding factors 
were not controlled. Some differences in baseline condition may not be detected by type II error. Second, the early dexamethasone group may have included more patients with less severe COVID-19 pneumonia, although there was no difference in SOFA scores and SAPS II between the early and late dexamethasone groups. The late dexamethasone group received dexamethasone only after progression of hypoxemia, whereas in the early group dexamethasone was administered as soon as hypoxemia occurred. Therefore, the patients with a milder disease, who improved without dexamethasone, may not have been included in the late dexamethasone group, but would have been included in the early dexamethasone group. Third, our study did not include very severe cases with rapid progression to ARDS. We excluded three patients who had severe hypoxemia at admission and needed HFNC treatment immediately, before dexamethasone was started. Although the anti-inflammatory effect of dexamethasone is likely to be most pronounced in ARDS patients with rapid progression, our results cannot be extrapolated to these patients. Fourth, the early and late dexamethasone groups had similar rates of remdesivir treatment; however, the timing of remdesivir administration was as late as that of dexamethasone in the late dexamethasone group.

In conclusion, the administration of dexamethasone within 24 hours of oxygen supplementation correlated with a lower rate of HFNC or MV treatment in patients with severe COVID-19 pneumonia. The duration of oxygen supplementation and length of hospital stay were shorter in the early dexamethasone treatment group. Early administration of dexamethasone may also be beneficial in the prevention of ARDS progression and may prevent the need for HFNC or MV treatment, without an increase in fatal events.

\section{Authors' Contributions}

Conceptualization: Lee HW, Heo EY. Formal analysis: Lee HW, Heo EY. Data curation: Lee HW, Heo EY. Writing - original draft preparation: Lee HW. Writing - review and editing: Park M, Lee JK, Park TY, Heo EY. Approval of final manuscript: all authors.

\section{Conflicts of Interest}

No potential conflict of interest relevant to this article was reported.

\section{Funding}

No funding to declare.

\section{Supplementary Material}

Supplementary material can be found in the journal homepage (http://www.e-trd.org).

Supplementary Table S1. Serial change of physiologic parameters from the time when hypoxemia occurred (day 0) to day 3.

\section{References}

1. WHO Rapid Evidence Appraisal for COVID-19 Therapies (REACT) Working Group; Sterne JA, Murthy S, Diaz JV, Slutsky AS, Villar J, et al. Association between administration of systemic corticosteroids and mortality among critically ill patients with COVID-19: a meta-analysis. JAMA 2020;324:1330-41.

2. RECOVERY Collaborative Group; Horby P, Lim WS, Emberson JR, Mafham M, Bell JL, et al. Dexamethasone in hospitalized patients with COVID-19. N Engl J Med 2021;384:693-704.

3. Tomazini BM, Maia IS, Cavalcanti AB, Berwanger O, Rosa RG, Veiga VC, et al. Effect of dexamethasone on days alive and ventilator-free in patients with moderate or severe acute respiratory distress syndrome and COVID-19: the CoDEX randomized clinical trial. JAMA 2020;324:1307-16.

4. Dequin PF, Heming N, Meziani F, Plantefeve G, Voiriot G, Badie J, et al. Effect of hydrocortisone on 21-day mortality or respiratory support among critically ill patients with COVID-19: a randomized clinical trial. JAMA 2020;324:1298-306.

5. Angus DC, Derde L, Al-Beidh F, Annane D, Arabi Y, Beane A, et al. Effect of hydrocortisone on mortality and organ support in patients with severe COVID-19: the REMAP-CAP COVID-19 Corticosteroid Domain Randomized Clinical Trial. JAMA 2020;324:1317-29.

6. Johnson RM, Vinetz JM. Dexamethasone in the management of COVID-19. BMJ 2020;370:m2648.

7. Villar J, Ferrando C, Martinez D, Ambros A, Munoz T, Soler JA, et al. Dexamethasone treatment for the acute respiratory distress syndrome: a multicentre, randomised controlled trial. Lancet Respir Med 2020;8:267-76.

8. Fadel R, Morrison AR, Vahia A, Smith ZR, Chaudhry Z, Bhargava $P$, et al. Early short-course corticosteroids in hospitalized patients With COVID-19. Clin Infect Dis 2020;71:2114-20.

9. Zhao Z, Zhang F, Xu M, Huang K, Zhong W, Cai W, et al. Description and clinical treatment of an early outbreak of severe acute respiratory syndrome (SARS) in Guangzhou, PR China. J Med Microbiol 2003;52:715-20.

10. von Elm E, Altman DG, Egger M, Pocock SJ, Gotzsche PC, Vandenbroucke JP, et al. The Strengthening the Reporting of Observational Studies in Epidemiology (STROBE) statement: guidelines for reporting observational studies. J Clin Epidemiol 2008;61:344-9.

11. Emanuel EJ, Persad G, Upshur R, Thome B, Parker M, Glick- 
man A, et al. Fair allocation of scarce medical resources in the time of COVID-19. N Engl J Med 2020;382:2049-55.

12. Annane D, Pastores SM, Arlt W, Balk RA, Beishuizen A, Briegel J, et al. Critical illness-related corticosteroid insufficiency (CIRCI): a narrative review from a Multispecialty Task Force of the Society of Critical Care Medicine (SCCM) and the European Society of Intensive Care Medicine (ESICM). Crit Care Med 2017;45:2089-98.

13. Huang C, Wang Y, Li X, Ren L, Zhao J, Hu Y, et al. Clinical features of patients infected with 2019 novel coronavirus in Wuhan, China. Lancet 2020;395:497-506.

14. Zhou Y, Fu B, Zheng X, Wang D, Zhao C, Qi Y, et al. Pathogenic T-cells and inflammatory monocytes incite inflammatory storms in severe COVID-19 patients. Nat Sci Rev 2020;7:9981002
15. Hojyo S, Uchida M, Tanaka K, Hasebe R, Tanaka Y, Murakami $\mathrm{M}$, et al. How COVID-19 induces cytokine storm with high mortality. Inflamm Regen 2020;40:37.

16. Merad M, Martin JC. Pathological inflammation in patients with COVID-19: a key role for monocytes and macrophages. Nat Rev Immunol 2020;20:355-62.

17. Meduri GU, Annane D, Chrousos GP, Marik PE, Sinclair SE. Activation and regulation of systemic inflammation in ARDS: rationale for prolonged glucocorticoid therapy. Chest 2009;136:1631-43.

18. Lee HW, Yoon SY, Lee JK, Park TY, Kim DK, Chung HS, et al. Clinical implication and risk factor of pneumonia development in mild coronavirus disease 2019 patients. Korean J Intern Med 2021;36:1-10. 